\title{
Dynamics of permeable particles in concentrated suspensions
}

\author{
Gustavo C. Abade, ${ }^{1}$ Bogdan Cichocki, ${ }^{1}$ Maria L. Ekiel-Jeżewska, ${ }^{2},{ }^{*}$ Gerhard Nägele, ${ }^{3}$ and Eligiusz Wajnryb ${ }^{2}$ \\ ${ }^{1}$ Institute of Theoretical Physics, University of Warsaw, Hoża 69, 00-681 Warsaw, Poland \\ ${ }^{2}$ Institute of Fundamental Technological Research, \\ Polish Academy of Sciences, Pawińskiego 5B, 02-106 Warsaw, Poland \\ ${ }^{3}$ Institut für Festkörperforschung, Forschungszentrum Jülich, D-52425 Jülich, Germany
}

(Dated: October 6, 2009)

\begin{abstract}
We calculate short-time diffusion properties of suspensions of porous colloidal particles as a function of their permeability, for the full fluid-phase concentration range. The particles are modeled as spheres of uniform permeability with excluded volume interactions. Using a precise multipole method encoded in the HYDROMULTIPOLE program, results are presented for the hydrodynamic function, $H(q)$, sedimentation coefficient, and self-diffusion coefficient with a full account of manybody hydrodynamic interactions. While self-diffusion and sedimentation are strongly permeability dependent, the wavenumber dependence of the hydrodynamic function can be reduced, by appropriate shifting and scaling, to a single master curve, independent of permeability. Generic features of the permeable sphere model are discussed.
\end{abstract}

Suspensions of solvent-permeable colloidal particles can be found in a great variety of synthesized materials. Examples are fuzzy-sphere systems consisting of highly porous, cross-linked microgel spheres exhibiting large volume changes as a function of temperature $[1,2]$. Another experimentally well-studied class of permeable colloids are core-shell-like particles consisting of an impermeable rigid core and a permeable stabilizing layer of some soft material [3, 4], such as grafted polymers [5, 6]. Despite the importance of permeable particles both from a fundamental viewpoint and in terms of applications, little is known theoretically about transport properties in non-dilute systems, such as self- and collective diffusion coefficients. The calculation of transport properties is a challenging problem since one has to cope with manybody hydrodynamic interactions (HIs) by accounting for the fluid flow inside the porous particles relative to their skeletons. A better control on diffusion and viscoelastic properties for industrial processing of concentrated colloids requires a deeper understanding of the influence of the HIs.

Theoretical and simulation work on diffusion and sedimentation of porous particles was primarily concerned so far with dilute systems. Chen and Cai [7] calculated the sedimentation velocity in a suspension of uniformly porous spheres to first order in the volume fraction $\phi$, demonstrating that sedimentation is quite sensitive to direct interactions and permeability. Mo and Sangani [8] used a multipole expansion method for hydrodynamically interacting porous spheres to obtain numerical results for the average drag force per particle in random and in bcc fixed-bed arrays.

Clearly, there is a strong demand on exploring generic HIs effects in concentrated porous particle systems where pairwise additivity approximations are bound to fail. In this letter, we describe a comprehensive simulation study of short-time diffusion properties for systems of permeable non-overlapping spheres. Our study covers the whole fluid-phase regime including concentrated systems with strong many-body HIs. The considered permeability range from fully impermeable to strongly permeable particles. Numerical results are presented for the hydrodynamic function, $H(q)$, obtained in short-time scattering experiments as a function of wavenumber $q$, and for the associated short-time self-diffusion coefficient, $D_{s}$, and mean sedimentation velocity $U$.

The function $H(q)$ is the key quantity containing information on colloidal short-time diffusion processes. In a dynamic scattering experiment, $H(q)$ is obtained from measuring the short-time exponential decay, $S(q, t) / S(q) \approx \exp \left[-q^{2} D(q) t\right]$, of the dynamic structure factor $S(q, t)$. The measured short-time diffusion function, $D(q)$, is used to define $H(q)$ by the relation [9]

$$
D(q)=D_{0} H(q) / S(q),
$$

where $S(q)=S(q, 0)$ is the static structure factor and $D_{0}$ is the diffusion coefficient of a single porous particle. In the limit of $q \rightarrow \infty, H(q)$ is equal to the normalized short-time self-diffusion coefficient, $D_{s} / D_{0}$. At $q \rightarrow 0$, $H(q)$ reduces to the sedimentation coefficient $K=U / U_{0}$, with the sedimentation velocity $U_{0}$ of a porous particle at infinite dilution.

The statistical-mechanical expression for $H(q)$ is [9]

$H(q)=\left\langle\frac{k_{B} T}{N D_{0}} \sum_{i, j=1}^{N} \exp \left[i \mathbf{q} \cdot\left(\mathbf{R}_{i}-\mathbf{R}_{j}\right)\right] \hat{\mathbf{q}} \cdot \boldsymbol{\mu}_{i j}(\mathbf{X}) \cdot \hat{\mathbf{q}}\right\rangle$,

where $\hat{\mathbf{q}}$ is the unit vector in direction of the scattering wavevector $\mathbf{q}$. The brackets represent an equilibrium ensemble average in the thermodynamic limit $N \rightarrow \infty$, with fixed density $n=N / V$. The translational mobility tensors $\boldsymbol{\mu}_{i j}(\mathbf{X})$ linearly relate the hydrodynamic forces acting on the spheres to their translational velocities. They depend on the hydrodynamic model of particles, and on the many-body HIs between them at the specific configuration $\mathbf{X}=\left\{\mathbf{R}_{1}, \cdots, \mathbf{R}_{N}\right\}$ of their centers. 
To focus on generic HIs effects caused by permeability using a minimal number of parameters, we model the particles as solvent-permeable, non-overlapping spheres of uniform permeability $k$, and radius $a$. The velocity field, $\mathbf{v}(\mathbf{r})$, and pressure field, $p(\mathbf{r})$, of the Newtonian fluid of shear viscosity $\eta_{0}$ outside an inside the spheres are described, respectively, by the Stokes [10] and Brinkman $[11,12]$ equations,

$$
\eta_{0} \boldsymbol{\nabla}^{2} \mathbf{v}-\eta_{0} \kappa^{2}\left[\mathbf{v}-\mathbf{u}_{i}\right] \chi(\mathbf{X})-\boldsymbol{\nabla} p=0, \quad \boldsymbol{\nabla} \cdot \mathbf{v}=0 .
$$

Here, $\kappa^{-1}=\sqrt{k}$ is the hydrodynamic screening length, and $\mathbf{u}_{i}(\mathbf{r})=\mathbf{U}_{i}+\boldsymbol{\omega}_{i} \times\left(\mathbf{r}-\mathbf{R}_{i}\right)$ is the rigid-body velocity field of the skeleton of a sphere $i$ moving with translational and rotational velocities $\mathbf{U}_{i}$ and $\boldsymbol{\omega}_{i}$, respectively. The characteristic function, $\chi(\mathbf{X})$, is equal to one for $\mathbf{r}$ inside a sphere and zero otherwise. The Brinkman equation obtained for $\chi=1$ describes the mean flow inside a porous particle, under the proviso that the mean pore size, $\delta \sim \kappa^{-1}$, is sufficiently smaller than $a$, i.e., the dimensionless inverse hydrodynamic screening length, $x=\kappa a$, is sufficiently large.

To determine the translational hydrodynamic mobility tensors $\boldsymbol{\mu}_{i j}(\mathbf{X})$ going into the calculation of diffusion properties, the Stokes equation for the flow outside the spheres (where $\chi=0$ ) and the Brinkman equation inside the spheres must be solved under the conditions that $\mathbf{v}$ and the fluid stress tensor change continuously across the sphere surfaces. Here we apply the multipole expansion $[13,14]$. In this procedure, two basic operators are involved: the integral operator $\mathbf{G}(i j)$ with the Green kernel equal to the Hasimoto tensor for a periodic system [15], describing the propagation of the velocity field from the particle $j$ to the particle $i$, and the onesphere operator, $\mathbf{Z}_{0}(i)$, which relates the incident flow around particle $i$ to the flow scattered by this particle. Only $\mathbf{Z}_{0}(i)$ depends on the hydrodynamic particle model, i.e., on whether porous particles [16], particles with stick boundary conditions or liquid droplets are considered. The operators $\mathbf{Z}_{0}(i)$ and $\mathbf{G}(i j)$ enter into a system of integral equations, which are solved numerically with the HYDROMULTIPOLE code, by projecting onto a complete set of spherical multipole functions, and truncating at a multipole order $L[13,14]$. We use $L=3$.

The averaging is performed over equilibrium configurations of typically $N=256$ particles in a periodically replicated cubic simulation cell. The configurations are generated by Brownian dynamics simulations and a condensation technique. The extrapolation from a finite number of particles in the periodic cell to the thermodynamic limit, $N \rightarrow \infty$, is achieved using a finite-size correction formula proposed by Ladd [17], and our fastmultipole simulations performed for $N=256,512,1024$. With the extrapolation for $N \rightarrow \infty$, and also the extrapolation for the truncation order $L \rightarrow \infty$, high-precision data for $H(q)$ are obtained, with an error of less than $1 \%$ at low $q$, and less than $0.5 \%$ at larger $q$.
The suspension in our model is fully characterized by the particle volume fraction $\phi$ and the parameter $x$. The former extends to values close to the freezing concentration, $\phi \leq 0.45$. The latter covers the whole range of permeability, $x \geq 3$, starting from highly porous particles at the limits of the continuum picture underlying the Brinkman equation, and including typical values $x \approx 30$, which correspond to screening lengths of polymer brushes in core-shell systems, e.g., $\kappa^{-1} \approx 5 \mathrm{~nm}$ and $a=140 \mathrm{~nm}$ for the particles studied in [6].

Fig. 1 shows an example of our simulation results for

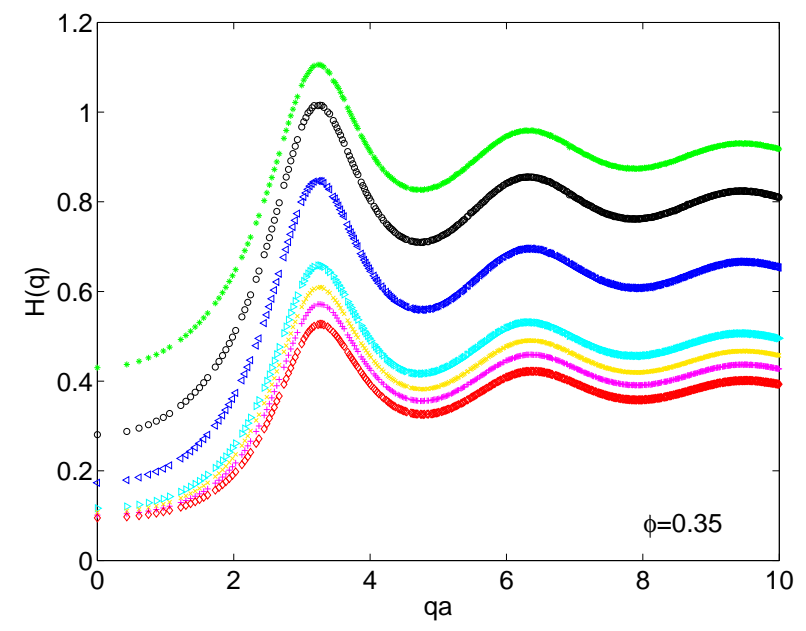

FIG. 1: Simulation results for the hydrodynamic function $H(q)$ at $x=3,5,10,30,50,100, \infty$ (from top to down).

$H(q)$ at $\phi=0.35$, covering a wide range of $x$ values. With increasing $x, H(q)$ becomes smaller at all $q$ [18]. The decrease reflects the strengthening of the HIs when the particles become less permeable. This can be understood if $H(q)$ is interpreted as the short-time generalized sedimentation coefficient of a homogeneous suspension of monodisperse particles subject to a weak, spatially sinusoidal force field collinear with $\mathbf{q}$ [19]. According to our simulations, the amplitude of oscillations in $H(q)$ becomes larger with increasing $\phi$.

Three characteristic values of the hydrodynamic function $H(q)$ are shown in Fig. 2: at the principal peak position $q_{m}$, at $q=0$ and for $q \rightarrow \infty$, representing $H\left(q_{m}\right)$, $K$ and $D_{s} / D_{0}$, respectively. The single-particle diffusion coefficient, $D_{0}=D_{0}(x)$, is very sensitive to $x[20]$. In the zero permeability limit, $x \rightarrow \infty$, it attains its smallest value given by the Stokes-Einstein diffusion coefficient, $D_{0}(\infty)=D_{0}^{\mathrm{hs}}=k_{B} T /\left(6 \pi \eta_{0} a\right)$, of a nonpermeable sphere with stick boundary conditions.

For $x \geq 20, H\left(q_{m}\right)$ decays monotonically in $\phi$, whereas a small non-monotonicity is observed for $x \approx 5$ (see Fig. $2 \mathrm{a}$ ). The peak value declines most strongly for zero permeability $(x=\infty)$, where it follows precisely the linear relation $H\left(q_{m}\right)=1-1.35 \phi$ up to $\phi=0.45$. This confirms earlier Stokesian dynamics simulation results for 

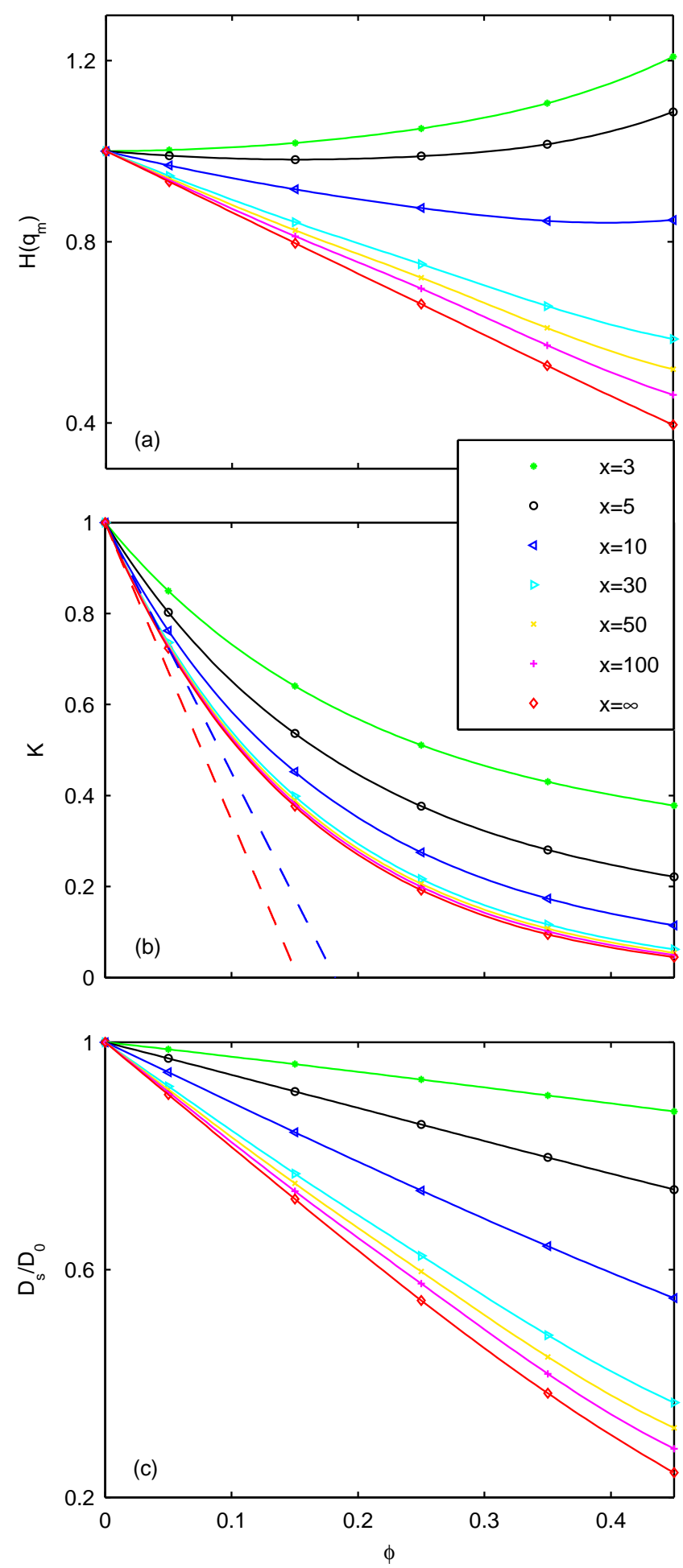

FIG. 2: Characteristic values of the hydrodynamic function $H(q)$, with simulation points connected by spline fits to guide the eye. (a) Principal peak value $H\left(q_{m}\right)$. (b) Sedimentation coefficient $K$. Dashed red and blue lines are the first-order concentration results for non-permeable hard spheres, $K^{\text {hs }}=$ $1-6.546 \phi[21]$, and highly permeable particles with $x=10$, $K=1-5.5 \phi[7]$. (c) Normalized self-diffusion coefficient. the $H\left(q_{m}\right)$ of impermeable hard spheres [22].

Unlike $H\left(q_{m}\right)$, both $K$ and $D_{s} / D_{0}$ decline monotonically for all $x$, with a faster decay for a smaller permeability (see Figs. 2b and c). The quantities in Fig. 2 are strongly permeability dependent, in particular at larger $\phi$. Decreasing $x$ from 100 to 10 at $\phi=0.45$, e.g., doubles $K$ and $D_{s} / D_{0}$. The dashed lines in Fig. $2 \mathrm{~b}$ are the firstorder density expansion results for $x=\infty$ and $x=10$ derived, respectively, by Batchelor [21] and Chen and Cai [7]. Notice that $\mathcal{O}\left(\phi^{2}\right)$ corrections to $K$ involving threebody HIs contribute significantly already for $\phi=0.05$.

The intra-particle flow is attenuated with increasing $x$ (decreasing permeability). The flow reduction enhances both the inter-particle hydrodynamic friction, so that $U$ and $D_{s}$ are decreased, and the single-particle hydrodynamic friction, so that also $U_{0}$ and $D_{0}$ are decreased. From Fig. 2 it is clear that the first effect is stronger, because $U / U_{0}$ and $D_{s} / D_{0}$ get smaller for increasing $x$.

In contrast to the strong dependence of $H\left(q_{m}\right), K$ and $D_{s} / D_{0}$ on the permeability, the principal peak location $q_{m}$ and the locations of the secondary maxima of $H(q)$ practically do not change with $x$ and are nearly coincident with those of $S(q)$. As a static equilibrium property, $S(q)$ is independent of hydrodynamics and thus of $x$. This observation leads to the central result of our study: while $H(q)$ itself is strongly permeability dependent, its $q$-dependence can be mapped essentially on that of impermeable hard spheres. To show this we introduce a reduced hydrodynamic function,

$$
h(q)=\frac{H(q)-H(\infty)}{|H(0)-H(\infty)|},
$$

where $H(\infty)=D_{s} / D_{0}$ and $H(0)=K$. Numerical results for $h(q)$ are shown in Fig. 3a.

Quite remarkably, for all wavenumbers $h(q)$ is nearly independent of $x$. This finding is valid for all $\phi=0.05-$ 0.45 . As a consequence, $h(q) \approx h^{\mathrm{hs}}(q)$, where $h^{\mathrm{hs}}(q)$ is the reduced hydrodynamic function of impermeable hard spheres dependent on $\phi$ only. Accordingly, $H(q)$ can be well-estimated in terms of the coefficients $K$ and $D_{s} / D_{0}$, and a $x$-independent master curve, $h^{\mathrm{hs}}(q)$. If the same reduction procedure is performed with $H(0)$ replaced by $H\left(q_{m}\right)$, the resulting function $h_{m}(q)$ coincides with a very high precision with $h_{m}^{\text {hs }}(q)$, if $q a>2$, as shown in Fig. 3b. For $q a<2, H(q)$ is a quadratic function of $(q a)^{2}$, with a non-negligible $(q a)^{4}$ contribution for $1 \leq q a \leq 2$.

Our simulations and a large- $q$ asymptotic analysis justify a suggestion by Pusey [23] that $D_{s} / D_{0}$ can be estimated by $H\left(q^{*}\right)$ at a wavenumber $q^{*}>q_{m}$ such that $S\left(q^{*}\right)=1$. According to our study, $D_{s} / D_{0}$ is obtained within the accuracy better than six percent for the first $q^{*}$ value to the right of $q_{m}$. A high precision value of $D_{s} / D_{0}$ with at most $1 \%$ deviation is obtained for the next larger $q^{*}$.

In summary, using a versatile simulation method, the short-time dynamic properties of uniformly permeable 


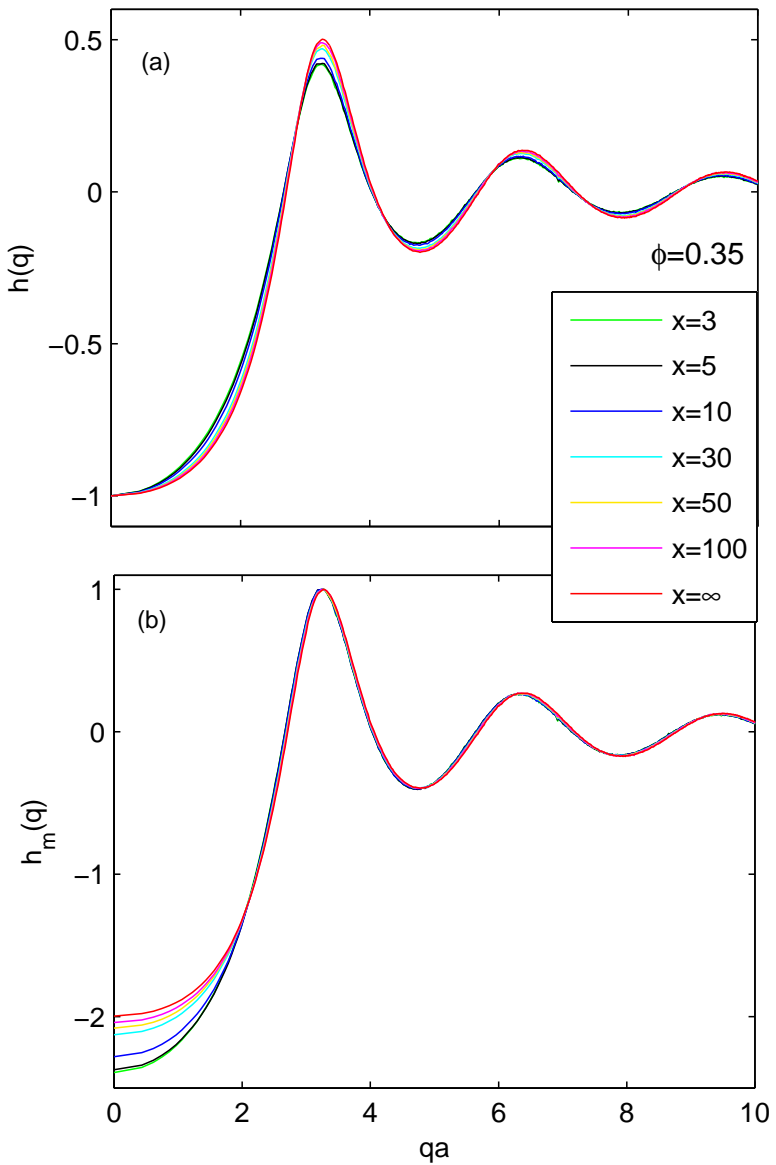

FIG. 3: Reduced hydrodynamic functions $h(q)$ and $h_{m}(q)=$ $[H(q)-H(\infty)] /\left[H\left(q_{m}\right)-H(\infty)\right]$.

spheres have been calculated as a function of permeability, concentration and wavenumber. Our most important result is that the $q$-dependence of $H(q)$ can be shifted and scaled to that of impermeable hard spheres, with the use of coefficients, which are strongly permeability dependent. The essential information about the hydrodynamic structure of particles is therefore contained only in the sedimentation, self-diffusion, and also cage-diffusion coefficients. Fig. 3 illustrates that any attempt to obtain transport properties of porous spheres using a model of impermeable effective hard spheres with an effective radius $a_{\text {eff }}$ rather than $a$, is not justified theoretically and will give unsatisfactory results.

The predictions of our study can be scrutinized in dynamic scattering experiments on systems of sterically stabilized porous particles. The generic trends for uniformly porous spheres are likely to apply also to hydrodynamically more complex particles such as the core-shell particles. The present data can serve as a database for experimentalists working on the dynamics of neutral porous colloidal particles and aggregates. Our work highlights the power and efficiency of the HYDROMULTIPOLE code in obtaining high-precision simulation data for dynamic properties of particles with internal hydrodynamic structure, for concentrated suspensions with strong manybody HIs.

G.N., M.L.E.J. and B.C. acknowledge support by the Deutsche Forschungsgemeinschaft (SFB-TR6, project B2). M.L.E.J. and E.W. were supported in part by the Polish Ministry of Science and Higher Education grant $45 / \mathrm{N}-\mathrm{COST} / 2007 / 0$ and the COST P21 Action "Physics of droplets". The work of G.C.A. was supported by CAPES Foundation/Ministry of Education of Brazil. Numerical calculations were done at the Academic Computer Center in Gdańsk, Poland. M.L.E.J. thanks Dirk van den Ende for helpful discussions.

* Electronic address: mekiel@ippt.gov.pl

[1] T. Eckert and W. Richtering, J. Chem. Phys. 129, 124902 (2008).

[2] E.H. Purnomo, D. van den Ende, S. A. Vanapalli, and F. Mugele, Phys. Rev. Lett. 101, 238301 (2008).

[3] G. Petekidis, J. Gapiński, P. Seymour, J. S. van Duijneveldt, D. Vlassopoulos and G. Fytas, Phys. Rev. E 69, 042401 (2004).

[4] M. Zackrisson, A. Stradner, P. Schurtenberger and J. Bergenholtz, Langmuir 21, 10835 (2005).

[5] H. M. Schaink, P. A. Nommensen, R. J. J. Jongschaap, and J. Mellema, J. Chem. Phys. 113, 2484 (2000).

[6] M. H. G. Duits, P. A. Nommensen, D. van den Ende, and J. Mellema, Colloids Surf. A 183-185, 335 (2001).

[7] S. B. Chen and A. Cai, J. Colloid Interface Sci. 217, 328 (1999).

[8] G. Mo and A. S. Sangani, Phys. Fluids 6, 1637 (1994).

[9] G. Nägele, Phys. Rep. 272, 215 (1996).

[10] S. Kim and S. J. Karrila, Microhydrodynamics, Dover Publications, Mineola (2005).

[11] H.C. Brinkman, Appl. Scient. Res. A1 27, 81 (1947).

[12] P. Debye and A.M. Bueche, J. Chem. Phys. 16, 573 (1948).

[13] B. Cichocki, B. U. Felderhof, K. Hinsen, E. Wajnryb, and J. Bławzdziewicz, J. Chem. Phys. 100, 3780 (1994).

[14] M.L. Ekiel-Jeżewska and E. Wajnryb, in Theoretical Methods for Micro Scale Viscous Flows, ed. by F. Feuillebois and A. Sellier, Transworld Research Network, Kerala (2009).

[15] H. Hasimoto, J. Fluid Mech. 5, 317 (1959).

[16] R. B. Jones and R. Schmitz, Physica A 149A, 373 (1988).

[17] A.J.C. Ladd, J. Chem. Phys. 93, 3484 (1990).

[18] G.C. Abade, B. Cichocki, M.L. Ekiel-Jeżewska, G. Nägele and E. Wajnryb, in preparation.

[19] A.J.C. Ladd, H. Gang, J.X. Zhu and D.A. Weitz, Phys. Rev. E 52, 6550 (1995).

[20] G. Neale, N. Epstein and W. Nader, Chem. Eng. Sci. 28, 1865 (1973).

[21] G.K. Batchelor, J. Fluid Mech. 52, 245 (1972).

[22] A.J. Banchio and G. Nägele, J. Chem. Phys. 128, 104903 (2008).

[23] P.N. Pusey, J. Phys. A 11, 119 (1978). 